\title{
Zeitschrift für Urologie. Band VI. Heft 7-9.
}

Waldschmidt, Max. Über Malakoplakia vesicae urinariae. Heft 7. pag. 541.

Zystoskopisch charakterisiert sich die Malakoplakie als eine mehr oder minder zahlreiche Aussaat gelblicher bis schwärzlicher Erhabenheiten, teils rund, teils mehr oval, einzeln stehend oder flächenhaft vereinigt, stecknadelkopf- bis markstückgroß, meist an der Oberfläche leicht usuriert, fast immer im Zentrum dellig eingesunken. Mikroskopisch sind charakteristisch Zelleinlagerungen, im und außerhalb des Protoplasmas liegend, ron verschiedener Form. Sie geben die Eisenreaktion. Mit Tannin-Orange und mit polychromem Methylenblau sind sie am besten darstellbar. $\mathrm{Ob}$ diese Finschlüsse Produlte phagozytärer oder ausscheidender Tätigkeit der die Knötchen bildenden Zellen sind, steht noch dahin.

Fast immer ist eine Sekundär-Infektion mit Koli-Bakterien bei dieser Krankheit vorhanden. Wenn auch ein erheblicher Anteil der beobachteten Fälle bei Individuen mit organ. Tuberkulose vorkam, ist doch die tuberkulöse Natur des Leidens so gut wie ausgeschlossen. Klinisch macht sich dasselbe kaum bemerkbar, wenigstens nicht in der Zeit vor der Koli-Infektion. Um wahre Geschwülste im histologischen Sinne handelt es sich nicht. Was die wahre Ursache ist, weiß man noch nicht.

Adrian, L, Zur Diagnose der Harnleiterfisteln. Heft 7. pag. 565 .

Neutralrot und Phenolsulfonephthaleïn geben, je nachdem sie in einem alkalischen oder saueren Medium gelöst beziehungsweise ausgeschieden worden, verschiedene Farben und kontrastreiche Farbveränderungen. Dieses Verhalten benützte Verf. zur Diagnose ron traumatisch (Geburt, Operationen im Abdomen) entstandenen Harngenitalfisteln beim Weibe, von angeborenen Mündungsanomalien derUreteren beim Weibe, von traumatischen Verletzungen undFisteln der Ureteren etc. Füllt man bei einer Harnleiterfistel die Blase z. B. mit schwach alkalischer Flüssigkeit $\left(\mathrm{NaHCO}_{3}\right.$ oder $\left.\mathrm{Na}_{2} \mathrm{CO}_{3}\right)$ und legt auf die Mündung der Fïstel einen Tampon mit Säure (z. B. 0.5\% Essigsäure), so wird bei Verwendung von Neutralrot $(0.01$ intramuskulär) der von den Nieren dunkelrot herabkommende Urin beim Eintritt in die Blase gelblich bis safrangelb, beim Zusammentreffen mit dem vor der Fistelmündung z. B. Vagina liegenden mit Essigsäure getränkten Tampon Aleischwasserfarben bis bordeauxrot, wenn die Fistel vom Ureter abgeht und nicht mit der Blase kommuniziert. Bei Phenolsulfonephthaleïn ist es umgekehrt. 
Zur Vermeidung von Fehlern und für die richtige Deutung der Resultate sind mancherlei Einschränkungen wohl zu beachten, die im Original nachzulesen sind.

Young, H. Eine neue Kpmbination von Zystoskop und Evakuations-Lithotrypter. Heft 7. p. 587.

Das neu konstruierte Instrument zeichnet sich dadurch aus, daß es ungewechselt Zertrümmerung des Steins, Evakuation der Trümmer und zystoskopische Nachuntersuchung der Blase nach zurückgebliebenen Trümmern erlaubt, dadurch Verletzungen der Harnröhre, Schmerzen wesentlich vermindert, so daß man mit Kokain-Anästhesie auskommt. Die gleichzeitige Evakuation, die die Trümmer in den Lithotrypter hineinzieht, beschleunigt die Operation und macht das wiederholte Absuchen der Blase überflüssig. Details der Konstruktion des Instruments im Original.

Orlowski. Der präneurotische Urin. Heft 7 . pag. 604 .

Wenn ein chronisch Harn- oder Geschlechtskranker durch seine Beschwerden oder die Behandlung genügend traktiert ist und sein Nervensystem darauf unliebsam zu reagieren droht, zeigt sich nach des Verf. Ansicht das drohende Unheil schon vorher durch eine Veränderung der normalen HarnaziditätsKurve an. Auch das relative Verbältnis des saueren und alkalischen Phosphats im Urin ist in dieser Beziehung, wenn auch nicht für sich allein, zu verwerten. Ferner ist der Indikangehalt des Urins eventuell von Bedeutung.

Denk, Wolfgang, Über Harnröhrendivertikel. Heft 8. pag. 621.

Die kongenitalen Divertikel sitzen meist im vordersten Teil der Pars cavernosa. selten weiter rückwärts; sie sind durch Stauung des Urins in der Harnröhre infolge verspäteter Kommunikation zwischen Penis- und Eichelharnröhre entstanden. Ihr Bau ist dem der vordersten Harnröhre ähnlich: papillenbildendes Bindegewebe mit mehrschichtigem Epithel ohne Verhornung. Die mit epidermisähnlichem Epithel ausgekleideten Divertikel sind wahrscheinlich erworbene. Infizierte Divertikel müssen inzidiert und sekundär geschlossen, nicht infizierte exzidiert und genäht werden, eventuell mit Faszienimplantation, oder es kommt die Resektion der Urethra in Betracht.

Lohnstein; H. Untersuchungen über die Länge der Pars posterior urethrae bei Urethritis und Urethroprostatitis. Heft 8. pag. 634.

Bei Urethritis posterior ist in der Regel die Pars posterior sehr verlängert und zwar infolge der Zunahme der Dimensionen - der Prostata (Prostatitis). Auch ohne Prostatitis fand Verf. 
eine Verlängerung bei allen Individuen, die älter als 40 Jahre waren (wenigstens $6 \mathrm{~cm}$ lang). Vorzugsweise streckt sich der Abschnitt zwischen Colliculus und Sphincter internus. Die obere Wand der Pars posterior hält nicht gleichen Schritt mit der unteren, je länger letztere, um so kleiner die Differenz. Die Seitenwände sind kürzer und unter sich auch von verschiedenen Dimensionen.

Krebs, G. Ut ber den praktisch-therapeutischen Wert des Zystopurius. Heft 8. pag. 654 .

Empfehlung des Mittels bei 7.ystitis (idiopathischer, gonorrhoischer und der bei Prostata-Hypertrophie) sowie bei Nierenerkrankungen.

Uffreduzzi, O. Die Behandlung der Hodenretention. Heft 9. pag. 728.

Allgemeine Grundsätze sind nicht möglich aufzustellen. Jeder Fall ist verschieden zu beurteilen. Außer den örtlichen Verhältnissen spielen die psychischen und sozialen Verbältnisse des Patienten eine Rolle. Besonders schwierig ist die Entscheidung, wie man vorgehen soll bei beiderseitiger Retention. Ist das Leiden einseitig und, wie so oft, mit Hernie kompliziert, so muß man bei noch nicht vollendeter Geschlechtsreife mit der Operation warten, da der Hoden in der Funktion leiden oder an späterem Deszensus durch Narben gehindert werden kann.

Ein Eingriff ist indiziert bei Einklemmung, bei nicht reponibler und leicht entzündlicher Hernie und bei Hydrozele, namentlich kommunizierender.

Für alle anderen Fälle ist Massage oder vorsichtiger Zug vorzuziehen.

Körperliche Austrengungen sind zu vermeiden, nie darf ein Bruchband getragen werden.

Das Normalverfahren ist die Radikaloperation des Bruches, anschließend der Versuch einer Orchiopexie.

Die Kastration ist nie notwendig.

Bei doppelseitiger Hodenretention ist jeder Eingriff zu unterlassen, wenn keine Komplikation da ist.

Die Lage der Hoden im Leistenkanal stört im allgemeinen ihre Entwicklung gar nicht. Daher hat eine Operation mit dieser Begründung keine Berechtigung.

Bei doppelseitiger Anomalie ist erst versuchsweise eine Seite zu operieren, und auch nur falls eine Komplikation (Hernie) besteht, denn die unvermeidlichen Verletzungen des Funiculus können die schon so wie so gefährdete Samen bildende Funktion vollends zerstören.

Trinkler, N. P. Ein seltener Fall von OrganVerdoppelung. Heft 9. p. 751. 
Ein 3jähriges Mädchen hatte 2 regelmäßig entwickelte Vaginen, zwei Paar Labia minora, 2fache Klitoris, 2 Urethralöffnungen, vielleicht 2 Harnblasen. Wahrscheinlich kommunizierte Rektum mit Vagina. Das Kind hatle ferner einen doppelten Dickdarm (rom Blinddarm anfangend); 2 Uteri, in deren einen (oder Vagina) wahrscheinlich der linke Mastdarm einmündete, denn das Kind entleerte per vaginam Kot. Das Kind wurde einer linksseitigen Mastdarmresektion unterworfen und war seitdem kontinent. Betrachtungen über die verschiedenen Möglichkeiten in Art und Folge der embryonalen Entwicklungsstörungen.

Orlowski. Über seltene reflektorische Folgezustände der Kollikulus-Hypertrophie. H. 9. p. 767.

In einem Falle hatte die Kollikulus-Hypertrophie eine aufund abschwankende Epididymitis bilateralis verursacht, die erst nach Kaustik dauernd schwand, in einem zweiten ein unerträgliches Brennen in der Harnröhre, dem erst nach Ätzen mit $20 \%$ Arg. nitr. und leichter Galvanokaustik abgeholfen wurde, in einem dritten eine Herzneurose, periodische Depressionszustände. Auch hier Heilung durch 2malige Kaustik. Eine 4. Beobachtung betraf wieder rein subjektive Beschwerden in der Blasengegend und in den Leisten. Erfolg der Kaustik ebenfalls prompt. Die für Behandlung mit Kaustik sich eignende Kollikulus-Hypertrophie ist Folge von Coitus interruptus und frustraner Libido, während Onanie kaum in Frage kommt. Fast nie fehlt das Symptom der Ejaculatio praecox und Nachlaß der Potenz. Manchmal folgt auf den Eingriff Retentio urinae. Dann muß katheterisiert werden und dadurch entsteht dann leicht Prostatitis und Epididymitis, manchmal auch Blutung.

Rudolf Krösing. (Stettin).

\title{
The British Journal of Dermatology.
}

\author{
September 1912.
}

Gray, A. M. H. Ulcus rodens, aufgetreten in einem Psoriasisherde in der Glutaealfalte. p. 325.

Die Patientin Grays, eine 56jährige Frau, litt seit ihrem 24. Jahre an Psoriasis, die trotz mannigfacher äußerer und innerer Behandlung - sie hat auch reichlich Arsenik bekommen - nie abgeheilt war. Seit 10 Jahren hatte sich aus einem Psoriasisberde der Glutaealfalte eine ausgedehnte ulzerierende Buldung entwickelt, die als Lupus diagnostiziert und mit Radium und Röntgenstrahlen behandelt wurde. Der Autor 